\title{
From computational modelling of the intrinsic apoptosis pathway to a systems-based analysis of chemotherapy resistance: achievements, perspectives and challenges in systems medicine
}

\author{
ML Würstle ${ }^{1,2}$, E Zink $^{1,2}, \mathrm{JHM} \mathrm{Prehn}^{1,2}$ and M Rehm ${ }^{*, 1,2}$
}

Our understanding of the mitochondrial or intrinsic apoptosis pathway and its role in chemotherapy resistance has increased significantly in recent years by a combination of experimental studies and mathematical modelling. This combined approach enhanced the quantitative and kinetic understanding of apoptosis signal transduction, but also provided new insights that systemsemanating functions (i.e., functions that cannot be attributed to individual network components but that are instead established by multi-component interplay) are crucial determinants of cell fate decisions. Among these features are molecular thresholds, cooperative protein functions, feedback loops and functional redundancies that provide systems robustness, and signalling topologies that allow ultrasensitivity or switch-like responses. The successful development of kinetic systems models that recapitulate biological signal transduction observed in living cells have now led to the first translational studies, which have exploited and validated such models in a clinical context. Bottom-up strategies that use pathway models in combination with higher-level modelling at the tissue, organ and whole body-level therefore carry great potential to eventually deliver a new generation of systems-based diagnostic tools that may contribute to the development of personalised and predictive medicine approaches. Here we review major achievements in the systems biology of intrinsic apoptosis signalling, discuss challenges for further model development, perspectives for higher-level integration of apoptosis models and finally discuss requirements for the development of systems medical solutions in the coming years.

Cell Death and Disease (2014) 5, e1258; doi:10.1038/cddis.2014.36; published online 29 May 2014

Subject Category: Cancer

Facts

- Mathematical modelling has provided mechanistic insight into cell death decisions during intrinsic apoptosis signalling by identifying network-level control functions that emanate from multi-protein interplay.

- Validated systems models of intrinsic apoptosis can accurately predict signal transduction in individual cell lines, kinetically and quantitatively.

- In translational studies, systems models of intrinsic apoptosis show potential for predicting tumour responsiveness to genotoxic chemotherapy.

\section{Open Questions}

- Can systems models that accurately predict the responsiveness of cell lines to targeted apoptosis inducers and sensitisers be validated clinically?
- Will we be able to develop systems-based diagnostic tools that in the future may contribute to the development of personalised medicine and innovative patient stratification?

- Can clinical research environments adapt to fulfil the needs of translational systems biology and systems medical research?

The intrinsic apoptosis pathway can be initiated by a multitude of diverse stress signals (Figure 1a), including DNA damage, ER stress, cytoskeletal destabilisation, kinase and proteasome inhibition. These stresses result in the transcriptional or post-translational activation or accumulation of $\mathrm{BH} 3-$ only proteins, pro-apoptotic members of the B-cell lymphoma-2 (Bcl-2) protein family. BH3-only proteins activate the proapoptotic proteins Bax and Bak and/or antagonise anti-apoptotic members such as Bcl-2, Mcl-1, Bcl-xL. Activated Bax and Bak can homo-oligomerise to form pores

\footnotetext{
${ }^{1}$ Department of Physiology and Medical Physics, Royal College of Surgeons in Ireland, Dublin, Ireland and ${ }^{2}$ Centre for Systems Medicine, Royal College of Surgeons in Ireland, Dublin, Ireland

*Corresponding author: M Rehm, Department of Physiology and Medical Physics, Royal College of Surgeons in Ireland, RCSI York House, York Street, Dublin 0002, Ireland. Tel: +353 1402 8563; Fax: +353 1402 2447; E-mail: mrehm @ rcsi.ie

Keywords: systems biology; systems medicine; apoptosis; cell death

Abbreviations: Apaf-1, apoptotic protease activation factor 1; Bak, Bcl-2 homologous antagonist/killer; Bax, Bcl-2 associated X; Bcl-2, B cell lymphoma protein 2; $\mathrm{Bcl}-\mathrm{xL}, \mathrm{B}$ cell lymphoma extra large; $\mathrm{BH} 3, \mathrm{Bcl}-2$ homology 3 domain; Bid, $\mathrm{BH} 3$ interacting-domain death agonist; ER-Stress, endoplasmic reticulum stress; IAP, inhibitor of apoptosis; Mcl-1, induced myeloid leukemia cell differentiation protein; MOMP, mitochondrial outer membrane permeabilization; PUMA, p53-upregulated modulator of apoptosis; RSK, ribosomal s6 kinase; Smac, second mitochondria-derived activator of caspases; XIAP, x-linked inhibitor of apoptosis protein

Received 26.10.13; revised 20.12.13; accepted 02.1.14; Edited by B Zhivotovsky
} 


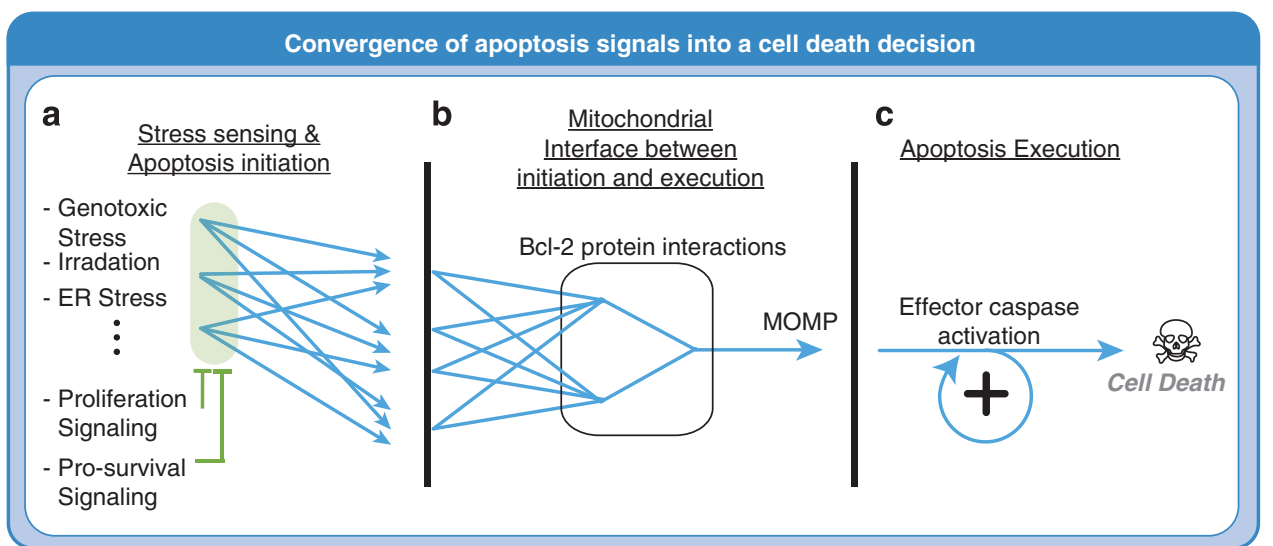

Figure 1 Convergence of complex apoptosis signals into a cell death decision - a simplified overview. (a) The upstream sensing of cellular stress and damage and the resulting initiation of apoptosis signalling is subject to a complex interplay of multiple signalling pathways. (b) Stress signals are integrated at the level of Bcl-2 family proteins. Their interactions and the regulation of their interactions dictate whether cells can undergo MOMP. (c) The apoptosis execution phase entails a number of positive feedback loops which result in the amplification of effector caspase activities and thereby ensures efficient cell death execution. Negative regulations in (b) and (c) were not visualised to increase clarity

in the outer mitochondrial membrane. Through these pores, the pro-apoptotic proteins cytochrome-c and Smac are released into the cytosol. The complex interactions of Bcl-2 family protein members therefore can integrate highly diverse upstream stress signals into a response of mitochondrial outer membrane permeabilisation (MOMP) (Figure 1b). In the cytosol, cytochrome-c triggers apoptosome formation and caspase-9 activation, whereas Smac antagonises caspaseinhibiting IAP proteins such as XIAP. Activated caspase-9 proteolytically activates effector caspases -3 and -7 . These effector caspases initiate feedback amplification loops and are the ultimate executioners of apoptotic cell death (Figure 1c). The execution phase is a largely dormant sub-system of apoptosis signal transduction, whereas the signalling network upstream of BH3-only protein signalling exists in a dynamic steady-state and continuously responds and adapts to stress signals.

Owing to the convergence of diverse stress signalling pathways into a common execution phase, and the fact that biochemical data of proteins and reactions involved in this phase have been made available, the execution phase has been the first target of systems biological studies that aimed to identify features, which determine and control cell death decisions. More recently, the first detailed systems approaches towards the understanding of $\mathrm{Bcl}-2$ protein interactions and MOMP have been developed. In contrast, the very early processes that initiate the intrinsic apoptosis pathway, such as the transcriptional control of $\mathrm{BH} 3-$ only protein production or their post-translational activation, have remained rather elusive or inaccessible to systems biological studies so far. An exception to this is the extrinsic apoptosis pathway that requires the post-translational activation of the BH3-only protein Bid (reviewed by Lavrik and colleagues in this special issue).

In the following, we will briefly describe the challenges faced for the systems biological analysis of early apoptosis initiation events and will then discuss and critically evaluate systems biological studies focusing on $\mathrm{Bcl}-2$ family protein interactions, MOMP regulation and apoptosis execution. Recent reviews on mathematical modelling techniques used in systems biological studies of apoptosis signalling may serve to obtain deeper methodological insight in this context. ${ }^{1-3}$ Open questions and important aspects to be considered for future systems-level investigations of apoptosis signalling will be covered in this context as well. We review studies that successfully applied apoptosis systems models in translational and clinical research settings, and will provide perspectives for the higher-level integration of apoptosis pathway modelling to allow multi-scale modelling beyond the single-cell level. Finally, we will highlight requirements and challenges faced for the clinical evaluation and implementation of apoptosis systems biology within the context of the newly emerging field of systems medicine.

\section{The Initiation of the Intrinsic Apoptosis Pathway - A Black Box for Kinetic Systems Modelling}

The initiation of the intrinsic pathway currently remains largely uncharted territory for kinetic systems modelling. This is partially owed to the diversity of stress signals that can initiate intrinsic apoptosis, the large number of $\mathrm{BH} 3$-only proteins, their possible combinations and their relative contributions towards signal transduction, and the respective mechanisms controlling the production or activation of individual BH3-only proteins (transcription, translation, post-translational modification). In addition, experimental tools that allow the collection of quantitative kinetic data for these processes at the singlecell level and which are required both for model parameterisation and for experimental validation of model predictions have not yet been developed. However, gene transcription and protein expression dynamics of other cellular signalling processes have been successfully monitored in real time in recent years. ${ }^{4-6}$ Such tools could therefore be adapted to investigate early stages of apoptosis initiation at the single-cell level. The need for a single-cell-based analysis is of particular importance for the initiation phase, as the majority of cellto-cell heterogeneities arise from variability in gene transcription and protein expression that affect early apoptosis signalling. ${ }^{7-9}$ 
Besides expression noise in apoptosis signalling proteins, interplays with non-apoptotic signalling pathways may also contribute significantly during early stages of signal transduction. For example, proliferation signalling is known to negatively regulate apoptosis susceptibility, and pathways involved in sensing and counteracting the damage inflicted by inducers of intrinsic apoptosis (e.g., genotoxic agents and ionising irradiation) contribute towards defining resistance towards apoptosis initiation. ${ }^{10,11}$ Large-scale statistical systems modelling building on complex data sets generated by high content and highthroughput experimental approaches rather than detailed kinetic pathway models therefore currently seem to be superior in describing and tackling the complexity of these pathway crosstalks. Indeed, such studies have successfully identified network components, which regulate and contribute towards defining apoptosis responsiveness. ${ }^{12-14}$

\section{Systems Analysis of the Regulation of MOMP}

During intrinsic apoptosis, MOMP is essential for triggering the apoptosis execution phase. MOMP is regulated by the family of Bcl-2 proteins. So far, more than a dozen members of this protein family have been identified, and these engage in a complex interplay of activation, inactivation and multimerisation. ${ }^{15}$ All Bcl-2 family members contain at least one of four different $\mathrm{Bcl}-2$ homology $(\mathrm{BH})$ domains $(\mathrm{BH} 1, \mathrm{BH} 2, \mathrm{BH} 3$ and $\mathrm{BH} 4)$, which define their functional role as either being pro- or anti-apoptotic. The anti-apoptotic Bcl-2 family members (Bcl-2, Mcl-1, Bcl-xL, A1 and Bcl-w) contain all four $\mathrm{BH}$ domains. They prevent apoptosis induction by antagonising the pro-apoptotic $\mathrm{Bcl}-2$ proteins. The pro-apoptotic fraction of the $\mathrm{Bcl}-2$ family can be divided into $\mathrm{BH}$ multi-domain proteins

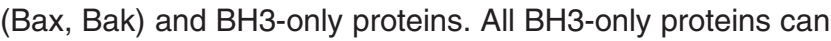
antagonise anti-apoptotic $\mathrm{Bcl}-2$ proteins, whereas the $\mathrm{BH}$ only proteins Bim, Puma and Bid can also directly activate Bax and Bak (Figure 2a). Individual BH3-only proteins or combinations thereof are induced or activated in response to stress signals that initiate the intrinsic apoptosis pathway, such as irradiation, genotoxic drugs or endoplasmic reticulum stress. Through Bax/Bak pores, pro-apoptotic factors are released into the cytosol to trigger apoptosis execution. Therefore, the interplay of $\mathrm{Bcl}-2$ family proteins that controls MOMP is highly complex.

Mathematical modelling studies of varying levels of complexity and detail have provided important insight into systems-emanating features that arise from $\mathrm{Bcl}-2$ proteins and have helped to mechanistically explain experimentally observed signalling characteristics. This includes the rapid switch-like kinetics of MOMP, the robustness of the ultrasensitive pore formation process to variability in protein expression levels, as well as the spatiotemporal spread of MOMP signals because of reaction-diffusion signalling. Such studies made use of kinetic models based on ordinary differential equations ${ }^{16,17}$ or spatial modelling approaches such as stochastic cellular automata simulations and partial differential equations based models. ${ }^{7,18,19}$ Following from initial systems theoretical investigations that studied the systems-level consequences, which arise from the known interaction topologies, these mathematical modelling approaches subsequently were successfully linked with quantitative experimental data.
Experimental observations of MOMP at the single-cell level demonstrated that the release of mitochondrial intermembrane space proteins is a rapid process that is completed within less than $5 \mathrm{~min} .{ }^{20}$ Only when investigated at a resolution of seconds, stimulus-specific differences in MOMP kinetics become apparent. ${ }^{7}$ Given that the overall duration of apoptosis signal transduction from initiation to execution typically requires many hours, the process of MOMP is a remarkably rapid unidirectional switch-like response of membrane permeabilisation. One of the most central systems biological problems therefore is to understand how this permeabilisation switch can emanate from the interplay of $\mathrm{Bcl}-2$ family proteins. In this context, experimentalists have discussed at length whether enabler and activator BH3-only proteins can drive rapid Bax pore formation equally efficiently (indirect versus direct activation scenario). In the indirect activation scenario, anti-apoptotic Bcl-2 family proteins antagonise active Bax or Bak proteins. Enabler BH3-only proteins interfere with this interaction and liberate active Bax and Bak by binding to anti-apoptotic Bcl-2 family members. In the direct activation scenario, the $\mathrm{BH} 3$-only protein members are divided into enabler and activator proteins. Although enabler proteins (such as Bad, Bmf, Noxa and Bik) only target anti-apoptotic $\mathrm{Bcl}-2$ family proteins, activator proteins (such as Bim, Puma and Bid) interact with and directly activate Bax and Bak, thereby triggering MOMP. Several systems theoretical studies investigated the plausibility of these two activation models. Chen et al. ${ }^{16}$ tackled this question long before conclusive experimental evidence was available, using a continuous deterministic modelling approach based on ordinary differential equations. A rigorously simplified interaction network considered only one species each of a Bax-like, a $\mathrm{Bcl}-2$-like and an enabler and activator $\mathrm{BH}$-only protein. ${ }^{16}$ Parameter screening showed that ultrasensitivity in pore formation (i.e., the rapid formation of large amounts of Bax pores upon reaching a critical threshold of $\mathrm{BH} 3-$ only proteins) is very unlikely to manifest from an indirect activation model. ${ }^{16}$ In contrast, a model of the direct activation scenario displayed ultrasensitive Bax pore formation processes for a large number of parameter sets. On the basis of this study, Bax pores would be expected to form in a switch-like manner once a critical input of activator BH3-only protein has accumulated. In contrast to the indirect activation model, the direct activation scenario furthermore is robust to perturbations in the respective protein amounts. ${ }^{16}$ These findings were largely validated in a recent study, which provided the so far most detailed mathematical model of the Bcl-2 interaction network, including a biologically meaningful quantitative parameterisation of protein amounts and rate constants ${ }^{21}$ (Figures $2 \mathrm{~b}$ and $\mathrm{c}$ ). In particular, it was shown that an indirect Bax/Bak activation mechanisms through enabler BH3-only proteins require strong antagonising regulatory mechanisms such as very high Bax/Bak pore degradation kinetics to prevent basal rates of spontaneous pore formation. ${ }^{21}$ In contrast, a direct activation scenario results in ultrasensitive pore formation responses that are robust to cell-to-cell heterogeneities in protein expression amounts and that were in line with previously published experimental data ${ }^{21}$ (Figure 2b). Integrating both activator and enabler $\mathrm{BH} 3-o n l y$ proteins in this model was sufficient to provide very good predictions on 
the MOMP susceptibility and cell death responsiveness of a number of cancer cell lines treated with genotoxic drugs ${ }^{17}$ (Figure 2c). The kinetics of Bax pore formation could also be reproduced qualitatively using a stochastic cellular automaton modelling, which represented Bax pore formation in membrane patches. ${ }^{18}$ This methodological approach was

\section{Bcl-2 protein signaling \& MOMP regulation}

a

$\mathrm{Bcl}-2, \mathrm{Bcl}-\mathrm{xL}, \mathrm{Mcl}-1, \ldots$

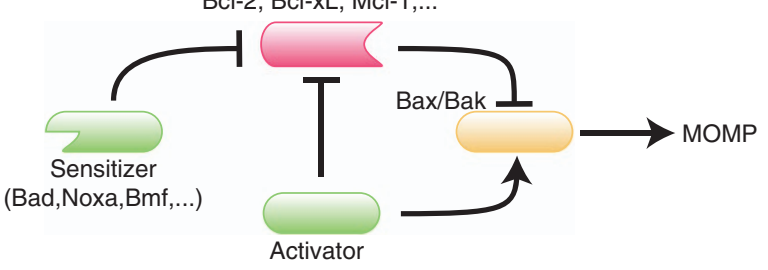

(tBid, Bim, Puma)

\begin{tabular}{l|l} 
Systems-level feature & Underlying molecular causes \\
\hline Ultrasensitivity/Molecular Switch & Cooperativity during Bax/Bak pore formation \\
\hline Robustness & Functionally redundant proteins
\end{tabular}

b direct activation model

indirect activation model

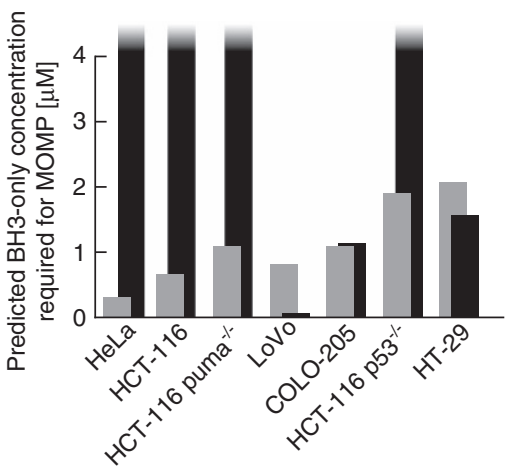

d

Measured number of mito. Bax molecules \pm SEM at onset of MOMP
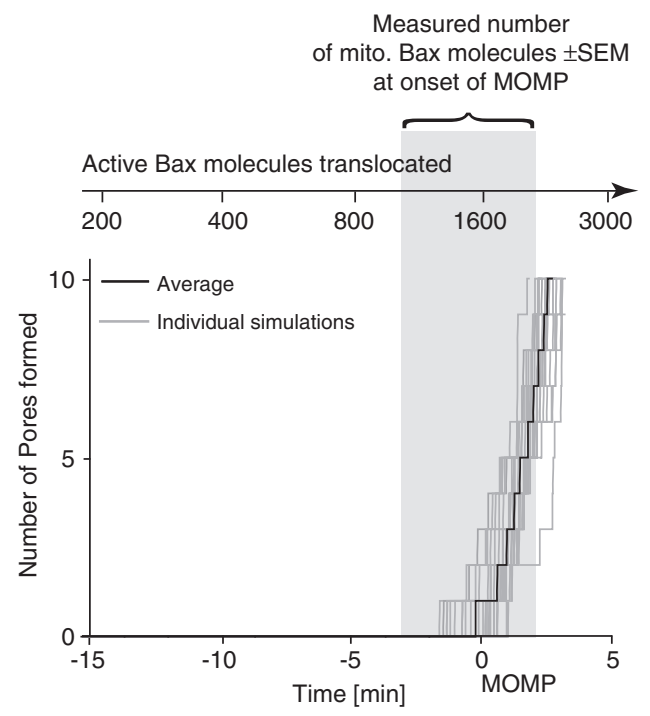

C

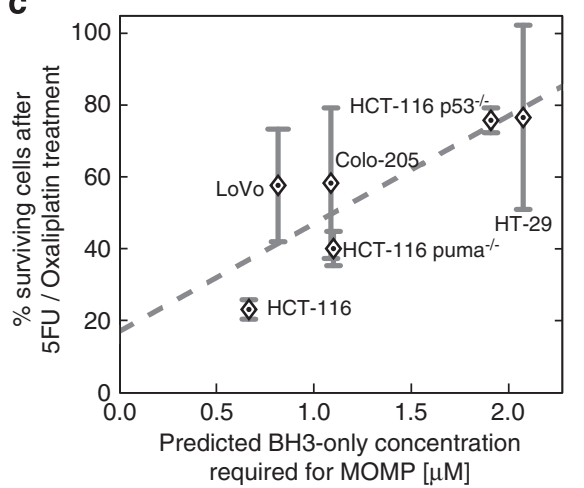

required for MOMP $[\mu \mathrm{M}]$ e

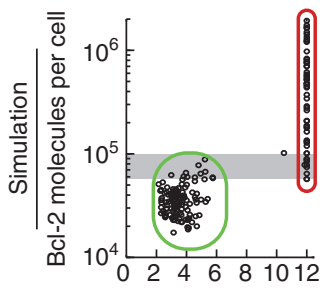
time to MOMP (hours) Bcl-2 Threshold

0 sensitive cells

insensitive cells

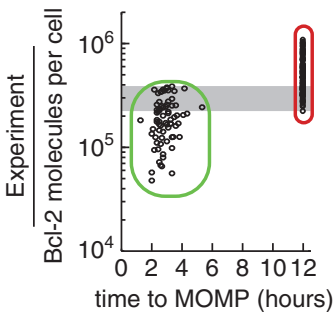


subsequently combined with quantitative experimental singlecell measurements in which absolute cytosolic and mitochondrial Bax quantities were measured in real time during MOMP. ${ }^{19}$ Not only could the mathematical model reproduce pore formation events at the experimentally measured amounts of mitochondrially accumulated Bax (Figure 2d), it was also found that only a small fraction of the entire Bax pool $(\sim 5 \%)$ needs to be activated in presence of activator and enabler BH3-only proteins to trigger pore formation. ${ }^{19}$ Mechanistically, it appears that the unidirectional switch-like process of MOMP emerges as a consequence of cooperative Bax oligomerisation, which occurs once a critical amount of active Bax has accumulated. ${ }^{19}$ MOMP then rapidly spreads through the cell body, a process that kinetically can be re-modelled by considering $\mathrm{BH} 3$-only proteins distributing spatiotemporally by reaction-diffusion processes. ${ }^{7}$ Interestingly, the velocity of these MOMP waves may depend on the rate of BH3-only protein production.

In agreement with the mathematical simulations, the importance of the direct activation mechanism for rapid pore formation has been supported experimentally by both mechanistic studies and by measurements of relative protein abundances of pro- and anti-apoptotic Bcl-2 family proteins. ${ }^{17,22,23}$ Nevertheless, to fully resemble the biological characteristics of MOMP, contributions from enabler $\mathrm{BH}$ only proteins towards Bax/Bak pore formation need to be taken into account as well. ${ }^{17,22,23}$ Although pore formation is an ultrasensitive, non-linear response, it needs to be noted that also the prevention of pore formation by anti-apoptotic $\mathrm{Bcl}-2$ proteins is controlled in a highly non-linear fashion. Gaudet et al. ${ }^{24}$ showed by both mathematical modelling and experimental measurements that critical thresholds of $\mathrm{Bcl}-2$ exist beyond which cells suddenly lose their capacity to undergo MOMP (Figure 2e). Importantly, this transition occurs across a narrow protein concentration range, and small cellto-cell differences in $\mathrm{Bcl}-2$ protein expression therefore can contribute towards explaining that only fractions of HeLa cell populations may respond to apoptotic signals. ${ }^{24}$

\section{Open Questions and Considerations}

The mechanistic and quantitative description of the individual interactions controlling Bax/Bak pore formation and MOMP kinetics is still far from complete. Especially, the complexity of the multiple interactions between the subfamilies of the $\mathrm{Bcl}-2$ proteins poses a challenge. Also, interactions appear to take place with significantly different reaction rates in aqueous and membranous environments, and contributions from non-Bcl-2 proteins may need to be considered as well. ${ }^{15,25-28}$ Additional studies aimed at determining binding characteristics of $\mathrm{Bcl}-2$ proteins at or in membranes are therefore required, and systems biological studies on the control of MOMP are likely to significantly benefit from such data and will allow a deeper biological understanding of MOMP regulation. Furthermore, current models have not taken into account the continuous cycling of $\mathrm{Bcl}-2$ proteins between the soluble cytosolic fraction and membrane-associated pools of the proteins, a process recently described for the $\mathrm{Bax} / \mathrm{Bcl}-\mathrm{xL}$ interplay. ${ }^{29,30}$ In addition, various $\mathrm{Bcl}-2$ proteins were shown to exhibit functions beyond apoptosis induction or prevention. For example, antiapoptotic $\mathrm{Bcl}-2$ proteins suppress autophagy by binding and inhibiting Beclin-1, a key protein required for early steps of autophagosome formation. ${ }^{31} \mathrm{Bcl}-2$ proteins also co-regulate mitochondrial fusion and fission as well as mitochondrial bioenergetics. ${ }^{32,33}$ Apoptosis systems modelling could therefore benefit from integrating such protein multi-functionalities as well as existing metabolic models in order to determine apoptosis susceptibility also in the context of cellular energy supply and mitochondrial fitness. ${ }^{34}$

\section{Systems Analysis of the Apoptosis Execution Phase}

As a consequence of MOMP, cytochrome-c and Smac are released into the cytosol. Cytochrome-c, together with dATP/ ATP, binds to monomeric Apaf-1. Apaf-1 then undergoes a conformational change and oligomerises into a stable heptamer that recruits procaspase- 9 . Procaspase- 9 is activated upon recruitment and proteolytically processed. The main procaspase- 9 substrates are the zymogens procaspase- 3 and -7 . Caspases $-9,-3$ and -7 can be inhibited by $\mathrm{XIAP}$, which itself is inhibited by Smac. Active caspase-3 is a key driver for rapid apoptosis execution by cleaving hundreds of cellular substrates and directly or indirectly causes the characteristic morphological changes associated with apoptotic cell death, such as cellular shrinkage, membrane blebbing and DNA fragmentation ${ }^{35,36}$ (Figure 3a). The kinetics of apoptosis execution resulting from such a linear sequence of events would be expected to be strongly sensitive to changes in the relative abundance of the involved proteins. However, real-time measurements in living cells demonstrated the opposite in that apoptosis execution in various cell lines with demonstrably different protein expression profiles proceed with nearly identical kinetics. ${ }^{9,37,38}$ Furthermore, substrate cleavage by effector caspases resembles an

Figure 2 Systems analysis of Bcl-2 protein signalling and MOMP. (a) Simplified overview of Bcl-2 protein interactions. Associated systems-level features and underlying molecular causes are listed. (b) Mathematical simulations of the calculated BH3-only protein amounts required to induce MOMP for scenarios of direct and indirect Bax/Bak activation. A number of cell lines known to be capable of undergoing MOMP would require excessive amounts of BH3-only proteins in an indirect activation setting. For the direct activation scenario, biologically justifiable amounts of BH3-only protein inputs were calculated for all cell lines, demonstrating that the direct activation model is in line with experimental evidence. Calculations were performed using kinetic models of Bcl-2 protein interactions. ${ }^{17,21}$ (c) Amounts of BH3-only proteins required for MOMP in a direct activation model were calculated for a number of colorectal cancer cell lines. Calculated $\mathrm{BH} 3-\mathrm{only}$ amounts correlate well with the responsiveness of the cell lines to genotoxic apoptosis inducers. Calculations were performed using a kinetic model of Bcl-2 protein interactions. ${ }^{17}$ (d) Stochastic simulations of Bcl-2 protein interactions and Bax pore formation in membrane patches. ${ }^{19}$ Modelling results indicate that the initiation of Bax pore formation correlates well with experimentally measured amounts of activated Bax at mitochondria. (e) Simulations of the effect of Bcl-2 protein expression amounts on the cellular competence to undergo MOMP resemble experimentally observed cellular behaviours. With increasing Bcl-2 protein amounts, cells undergo a sharp transition from MOMP competent to MOMP resistant. Simulations were conducted using a kinetic model of apoptosis signal transduction. ${ }^{24}$ Display items in panels (b-e) were reproduced or modified from previous publications ${ }^{17,19,21,24}$ 
all-or-none process, which is independent of the stimulus and stimulus strength and which is triggered by rapid effector caspase activation. ${ }^{9}$ The robustness of the substrate cleavage switch is established by amplifying feedback loops within the apoptosis execution network. ${ }^{39}$ The main positive feedbacks are the caspase-3-mediated removal of the

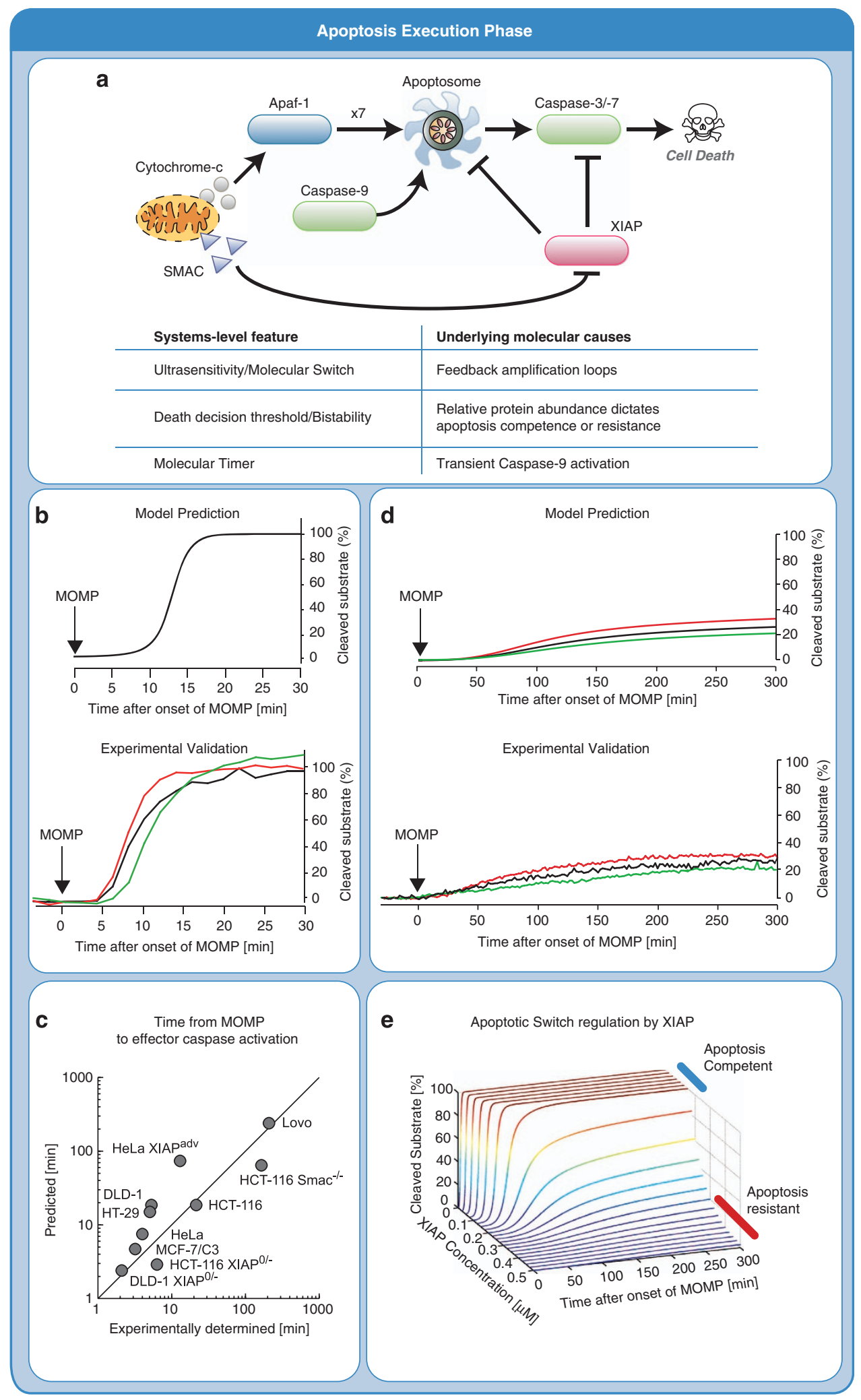


IAP-binding domain of caspase-9, which efficiently alleviates the inhibition of caspase- 9 by XIAP, ${ }^{40}$ and the sequestration of XIAP away from active caspase- 9 by active caspase- $3 .{ }^{41}$ The latter positive feedback manifests prominently when the amount of caspase-3 exceeds that of caspase- 9 and XIAP, as at these conditions activated caspase- 3 can serve as a sink that depletes XIAP and thereby re-activates caspase-9. It was also shown that caspase-3 may process and activate its own pro-form, albeit with significantly lower catalytic activity. ${ }^{42}$ A detailed mathematical model based on ordinary differential equations was developed to investigate the execution signalling network in HeLa cervical cancer cells. ${ }^{39}$ Parameterised with experimentally determined protein amounts, the mathematical simulations matched the experimentally recorded kinetics of effector caspase-dependent substrate cleavage very accurately (Figure $3 b$ ). Simulations were also sufficiently accurate to predict the time required to activate effector caspases in various other widely used cell line systems, including colorectal and breast cancer cell lines ${ }^{38}$ (Figure 3c). The in silico analysis of the consequences of altering the amounts of procaspase-9, Smac, Apaf-1 and procaspase-3 in HeLa cells indicated that the execution network remains functional and assured effector caspase activation across a wide range of protein concentrations. However, further analysis showed that the elevation of XIAP above a critical threshold concentration would result in a sudden collapse of the all-or-none response and at such conditions promote a limited and sublethal activation of effector caspases ${ }^{39}$ (Figure 3d). Importantly, the transition between efficient and sublethal induction of the apoptosis execution phase occurs across a narrow range of XIAP concentrations, indicating that modulations of XIAP amounts can transfer the network from an apoptosis competent to an apoptosis resistant state (Figure 3e). A systems theoretical investigation of the signalling network also identified that the execution network presents bi-stability characteristics in which the system can undergo a transition from a 'live' state to an irreversible state of lethal effector caspase activation. ${ }^{41}$ It is important to note that the switch-like transition from apoptosis competent to resistant is not a function of XIAP alone but rather emanates from the interplay of multiple network components. This transition is physiologically relevant and has been observed during the differentiation of post mitotic cells. For example, neurons and cardiomyocytes become apoptosis resistant by re-adjusting the relative abundance of execution network proteins during differentiation. ${ }^{43-45}$ Subsequent to quantitative kinetic simulations of apoptosis execution during intrinsic apoptosis, these signalling processes were also integrated successfully into models of extrinsic MOMP-dependent apoptosis signalling. ${ }^{46}$

\section{Open Questions and Considerations}

Although experimentally validated models of apoptosis execution have been remarkably accurate for the investigated cell line systems, they are of limited detail concerning the early molecular processes following MOMP. Especially, the formation of the Apaf- $1^{*}$ caspase- 9 apoptosome and caspase- 9 activation so far have been implemented mostly as input functions that remodel the experimentally determined in vitro kinetics of apoptosome formation and caspase- 9 activation. Although separate theoretical investigations into apoptosome formation were conducted, these only superficially integrated the available information on apoptosome formation mechanisms. ${ }^{47,48}$ A more detailed systems-level analysis would also be required to investigate and understand the potency of processes that were recently described to suppress Apaf-1 heptamerisation and caspase-9 activation. For example, phosphorylation of Apaf-1 by $90-\mathrm{kDa}$ ribosomal S6 kinase (RSK) prevents Apaf-1 oligomerisation. ${ }^{49}$ RSK is activated by the mitogen-activated protein kinase pathway, which is frequently constitutively (over-)activated in cancer cells. ${ }^{50}$ If Apaf-1 is phosphorylated by RSK, it recruits the adaptor protein $14-3-3 \varepsilon$, and $14-3-3 \varepsilon$ then blocks the binding of cytochrome-c, Apaf-1 activation and apoptosome formation. ${ }^{49}$ Similarly, Cdc6, a AAA + ATPase that is upregulated in various cancers and linked to carcinogenesis, ${ }^{51-53}$ prevents apoptosome formation by binding to cytochrome-c-activated Apaf-1. ${ }^{54}$ Of note, caspase-3 cleaves and inactivates Cdc6 during apoptosis execution. ${ }^{55}$ This feedback could free Apaf-1 from Cdc6-dependent inhibition and may restore apoptosome formation and thereby could enhance effector caspase activation. An extension of existing mathematical models could therefore serve to identify the context-dependent potencies of these mechanisms in preventing apoptosis, and the likelihood and robustness of inefficient and sub-lethal caspase activation. Current models also do not take into account that the apoptosome acts as a proteolytic-based molecular timer. ${ }^{56,57}$ Once procaspase- 9 is activated at the apoptosome, it undergoes auto-processing. Owing to its weak association with the apoptosome backbone, processed caspase- 9 is released into the cytosol and thereby becomes inactivated. At which conditions this molecular timer substantially contributes to cell death decisions has not been studied so far.

Owing to multi-protein reaction-diffusion processes during apoptosis execution, spatial anisotropies observed during the upstream event of MOMP are efficiently eliminated during apoptosis execution in epithelial cells, as was shown by theoretical and experimental analysis. ${ }^{58}$ However, spatially coordinated and limited apoptosis execution has

\footnotetext{
Figure 3 Systems analysis of the apoptosis execution phase. (a) Simplified overview of core signalling processes during the apoptosis execution phase. Associated systems-level features and underlying molecular causes are listed. (b) Kinetic simulations of substrate cleavage by effector caspases correlate well with experimental measurements in individual parental HeLa cells. ${ }^{39}$ (c) Mathematical calculations of the time required from MOMP until effector caspases activation in different cancer cell lines correlate well with experimental measurements. ${ }^{38}$ (d) Predictions on the consequence of XIAP overexpression on substrate cleavage by effector caspases were experimentally confirmed by measurements in HeLa cells overexpressing XIAP. Substrate cleavage is prolonged, submaximal and sublethal. ${ }^{39}$ (e) Mathematical analysis of the consequences of increasing XIAP amounts on effector caspase activity in HeLa cells. The simulations highlight that cells can undergo a sudden transition from apoptosis competent to apoptosis resistant once a critical XIAP concentration is exceeded. Display items in panels (b-e) are results from kinetic systems models of apoptosis execution and were reproduced or modified from previous publications ${ }^{38,39}$
} 
been observed in neuronal cells. ${ }^{59,60}$ A spatiotemporal systems analysis for such large, outstretched cells may therefore be required to understand whether our current knowledge on the reaction-diffusion properties of apoptosis execution proteins can explain the experimentally observed features of localised activation of effector caspases.

\section{Translational Application of Systems Models of the Intrinsic Apoptosis Pathway}

Although defects in apoptosis signalling are a hallmark of cancer, ${ }^{61}$ associating the expression of individual apoptosis proteins with cancer therapy resistance so far largely failed. It is therefore hoped that pathway systems models, which take into account multi-protein interactions, may be superior predictors for the responsiveness of cancer tissues to apoptosis-inducing treatments. First proof-of-concept studies that evaluated the translational capacity of apoptosis systems models have been conducted in recent years. Mathematical modelling of the Bcl-2 family network, parameterised with protein expression amounts found in colorectal cancer and matched normal tissue, identified that tumour tissue seems to be 'primed' and more likely to undergo apoptosis. ${ }^{17}$ Similar priming effects were previously identified experimentally in leukaemias and ovarian cancers by quantifying the effects of pro-apoptotic BCL-2 peptides on MOMP and mitochondrial depolarisation. ${ }^{62,63}$ Mathematical modelling also allowed to confidently identify those colorectal cancer patients that responded to 5-FU-based adjuvant treatment within the overall patient cohort. ${ }^{17}$ Similar translational potential was demonstrated by modelling the competence to activate effector caspases and therefore to execute apoptosis in stage 2/3 colorectal cancer patients receiving 5-FU-based adjuvant therapy. ${ }^{64}$ Tumour tissue in the majority of patients not responding to genotoxic chemotherapy appeared incapable of undergoing apoptosis execution. Similar results were recently also reported in patients suffering from glioblastoma multiforme, where results from the mathematical modelling of the competence of tumour cells to execute apoptosis correlated with progression-free survival times. ${ }^{65}$ Protein expression patterns investigated mathematically in the context of their pathway interplay could therefore serve as prognostic or predictive combinatorial systems biomarkers, ${ }^{17,64-66}$ and could provide an alternative when classical biomarker identification strategies are unsuccessful. ${ }^{67}$ Importantly, for scenarios in which apoptosis is blocked, a systems-level analysis can identify the functional causes for cell death resistance and therefore can identify strategies and intervention points that re-establish apoptosis competence. The feasibility of this concept has been demonstrated in recent proof-of-concept studies in which individualised predictions on the efficacy of apoptosis-sensitising cotreatments, such as BH3-mimetics or IAP antagonists, have been generated by modelling platforms. ${ }^{17,64,66}$ Taken together, such approaches therefore could provide the foundation for the development of a new generation of systems-based predictive and prognostic tools that in the future may contribute to the personalisation of treatments and may allow for patient stratification as part of innovative clinical trial design.

\section{Perspectives for the Integration of Apoptosis Pathway Models into Higher-Level and Multi-Scale Modelling Frameworks}

A major challenge for the further development and application of apoptosis pathway models is their integration into higherlevel contexts that reflect organisational structures, processes and interactions that go beyond the single-cell level (Figure 4). Signalling processes that occur in parallel or subsequent to apoptosis signal transduction and that directly or indirectly can affect cell death responsiveness may need to be considered. For example, the interplay of cellular and multi-cellular signalling relevant during cancer immunotherapies may need to be considered. ${ }^{68}$ Also, fractions of cells dying by nonapoptotic processes may contribute towards determining overall treatment efficacy, ${ }^{69}$ and these may also significantly alter the tumour microenvironment by promoting immune infiltration. Furthermore, activated caspase-3 released from dying cells may stimulate growth in surviving tumour cells and thereby can drive tumour repopulation, ${ }^{70}$ a function that could be related to the non-apoptotic role of caspase-3 in promoting wound healing through prostaglandin $\mathrm{E}_{2}$ signalling. ${ }^{71}$ Including such paracrine effects and interactions with surrounding cells and tissues may therefore be important to assess risks for tumour relapse and to more accurately predict short- and long-term clinical responses to apoptosis-inducing treatments. Similarly, the hierarchic organisation of certain tumour types, in which cancer stem cells may represent a distinct subpopulation that exists independent of stochastic cell-to-cell differences in gene or protein expression in the overall tumour cell population ${ }^{72,73}$ may need to be considered to accurately model and simulate tumour growth, invasion and therapy response over time. Initial studies in this direction were recently conducted and provide data on the behaviour of cell populations. ${ }^{74,75}$ As these population-based models are not yet linked with intracellular signalling pathways of apoptosis, this may provide an attractive avenue for future research.

The inclusion of pharmacokinetic (PK) and pharmacodynamic data (PD) and associated existing PK/PD models provides immediate opportunities for higher-level integration. Models capable of calculating drug penetration and resulting

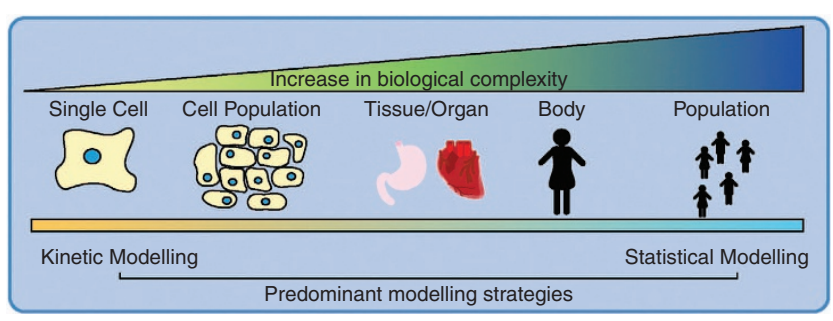

Figure 4 Systems modelling in the context of biological scale and complexity. Biological scale, biological complexity and the associated data availability and data quality influence systems modelling strategies. The most insightful single-cell and subcellular models of signalling pathways are based on kinetic simulations of reaction networks. The complexity of large-scale and population-level data sets are mostly tackled by statistical modelling approaches 
concentration profiles may be of particular importance to accurately replicate spatiotemporal profiles of apoptosisinducing stimuli in solid tumours. In this context, tumour vascularisation and its modulation by angiogenesis inhibitors may also need to be given special attention. First solutions and modelling frameworks that allow multi-scale integration have now been developed and can be applied in proof-of-concept studies. ${ }^{76}$ Furthermore, higher-level statistical modelling approaches that integrate patient clinical data as well as early treatment response data have already been used for personalising and optimising treatment schedules for chemo- and immunotherapies. ${ }^{77,78}$ It is also hoped that ultimately the higher-level integration of pathway models will enable complex and meaningful mathematical simulations of individual patients and patient cohorts before entering clinical trials (Figure 4). Such virtual trials could contribute towards assessing treatment efficacy a priori and may reduce the currently unsustainably high failure rate of phase II/III clinical trials. ${ }^{79-81}$ In addition, the need for predictive tools also exists for currently approved drugs and treatment regimens, as reliable personalised response predictions would reduce the number of unnecessary treatments and associated treatment costs.

\section{Future Challenges and Requirements for (Apoptosis) Systems Biology and Systems Medicine}

A general challenge both for basic and translational systems biological research of cell death processes is the lack of quantitative data, ${ }^{82}$ in particular at the level of protein expression, protein activities, interactions and turnover rates. Although several studies made efforts to accurately measure the expression amounts of apoptosis-relevant proteins in widely used cell line systems, ${ }^{17,38,39,66,83,84}$ the determination of protein quantities lacks commonly accepted and established methodological standards and may therefore be subject to considerable heterogeneity. In the clinical context, protein quantity measurements are even less common. Routine immunohistochemical analysis in pathology laboratories is limited by low dynamic range and usually not calibrated against samples with known protein content. Alternative methods that make use of tissue protein extracts, such as reverse-phase protein arrays, can provide quantities, but lack information on cell-to-cell heterogeneity within the tissue context and cannot be used in the routine setting. Clinical protein profiling techniques such as multiplex-ELISA will require further development to fulfil the needs of systems biological and systems medical studies. Although larger biobanking initiatives are now starting to widely collect tissue for subsequent analysis in research projects, sample storage and processing is not uniformly standardised, and the available amount of samples are often very limited. This is of particular concern regarding the collection of metastatic tumour tissue, as most novel therapeutics are initially tested in the metastatic setting. Limitations could be alleviated by a more comprehensive and earlier integration of clinician scientists into the design and coordination of translational systems biological research activities, and by the development of novel protein profiling techniques that can be integrated into clinical workflows. ${ }^{85}$ Furthermore, efforts need to be made to disseminate and communicate progress in the field of systems biology and systems medicine to medical professionals, to expert reviewers of clinical trial protocols and to patients and patient advocacy groups. Only in such an educated environment the transition from mathematical and experimental systems biology towards applied and validated novel systems medical approaches can be successful. The rapid progress and promising achievements in the field of apoptosis systems biology and apoptosis sensitising therapeutics may serve as a 'raw model' to facilitate this in the coming years.

\section{Conflict of Interest}

The authors declare no conflict of interest.

Acknowledgements. The authors kindly acknowledge support for their work by grants from the European Union (FP7 APO-DECIDE) and the Irish Health Research Board (APO-COLON; TRA/2007/26) to JHMP, and from Science Foundation Ireland (09/RFP/BIC2375) and an RCSI SFS Award (1671) to MR. We thank Nature Publishing Group, the American Society for Biochemistry and Molecular Biology, the Public Library of Science, the American Association for Cancer Research and the Royal Society of Chemistry for kind permission to re-use and modify display items presented in Figures 2 and 3.

1. Janes KA, Yaffe MB. Data-driven modelling of signal-transduction networks. Nat Rev Mol Cell Biol 2006; 7: 820-828.

2. Huber HJ, Bullinger E, Rehm M. Systems biology approaches to the study of apoptosis. In: Dong Z, Yin X-M (eds) Essentials of Apoptosis. Humana Press: New York, USA, 2009. pp 283-297.

3. Rehm M, Prehn JH. Systems modelling methodology for the analysis of apoptosis signal transduction and cell death decisions. Methods 2013; 61: 165-173.

4. Ashall L, Horton CA, Nelson DE, Paszek P, Harper CV, Sillitoe K et al. Pulsatile stimulation determines timing and specificity of NF-kappaB-dependent transcription. Science 2009; 324: 242-246.

5. Loewer A, Batchelor E, Gaglia G, Lahav G. Basal dynamics of p53 reveal transcriptionally attenuated pulses in cycling cells. Cell 2010; 142: 89-100.

6. Yu J, Xiao J, Ren X, Lao K, Xie XS. Probing gene expression in live cells, one protein molecule at a time. Science 2006; 311: 1600-1603.

7. Rehm M, Huber HJ, Hellwig CT, Anguissola S, Dussmann H, Prehn JH. Dynamics of outer mitochondrial membrane permeabilization during apoptosis. Cell Death Differ 2009; 16: 613-623.

8. Spencer SL, Gaudet S, Albeck JG, Burke JM, Sorger PK. Non-genetic origins of cell-to-cell variability in TRAIL-induced apoptosis. Nature 2009; 459: 428-432.

9. Rehm M, Dussmann $\mathrm{H}$, Janicke RU, Tavare JM, Kogel D, Prehn JH. Single-cell fluorescence resonance energy transfer analysis demonstrates that caspase activation during apoptosis is a rapid process. Role of caspase-3. J Biol Chem 2002; 277: 24506-24514.

10. Galluzzi L, Senovilla L, Vitale I, Michels J, Martins I, Kepp O et al. Molecular mechanisms of cisplatin resistance. Oncogene 2012; 31: 1869-1883.

11. Holohan C, Van Schaeybroeck S, Longley DB, Johnston PG. Cancer drug resistance: an evolving paradigm. Nat Rev Cancer 2013; 13: 714-726.

12. Janes KA, Albeck JG, Gaudet S, Sorger PK, Lauffenburger DA, Yaffe MB. A systems model of signaling identifies a molecular basis set for cytokine-induced apoptosis. Science 2005; 310: 1646-1653.

13. Lee MJ, Ye AS, Gardino AK, Heijink AM, Sorger PK, Macbeath G et al. Sequential application of anticancer drugs enhances cell death by rewiring apoptotic signaling networks. Cell 2012; 149: 780-794.

14. Janes KA, Gaudet S, Albeck JG, Nielsen UB, Lauffenburger DA, Sorger PK. The response of human epithelial cells to TNF involves an inducible autocrine cascade. Cell 2006; 124: 1225-1239.

15. Shamas-Din A, Kale J, Leber B, Andrews DW. Mechanisms of action of Bcl-2 family proteins. Cold Spring Harbor Perspect Biol 2013; 5: a008714.

16. Chen C, Cui J, Zhang W, Shen P. Robustness analysis identifies the plausible model of the Bcl-2 apoptotic switch. FEBS Lett 2007; 581: 5143-5150.

17. Lindner AU, Concannon CG, Boukes GJ, Cannon MD, Llambi F, Ryan D et al. Systems analysis of $\mathrm{BCL} 2$ protein family interactions establishes a model to predict responses to chemotherapy. Cancer Res 2013; 73: 519-528.

18. Chen C, Cui J, Lu H, Wang R, Zhang S, Shen P. Modeling of the role of a Bax-activation switch in the mitochondrial apoptosis decision. Biophys $J$ 2007; 92 : 4304-4315. 
19. Dussmann H, Rehm M, Concannon CG, Anguissola S, Wurstle M, Kacmar S et al. Single-cell quantification of Bax activation and mathematical modelling suggest pore formation on minimal mitochondrial Bax accumulation. Cell Death Differ 2010; 17: 278-290.

20. Goldstein JC, Waterhouse NJ, Juin P, Evan GI, Green DR. The coordinate release of cytochrome $\mathrm{C}$ during apoptosis is rapid, complete and kinetically invariant. Nat Cell Biol 2000; 2: 156-162.

21. Lindner AU, Prehn JH, Huber HJ. The indirect activation model of mitochondrial outer membrane permeabilisation (MOMP) initiation requires a trade-off between robustness in the absence of and sensitivity in the presence of stress. Mol Biosystems 2013; 9: 2359-2369.

22. Merino D, Giam M, Hughes PD, Siggs OM, Heger K, O'Reilly LA et al. The role of BH3-only protein Bim extends beyond inhibiting Bcl-2-like prosurvival proteins. J Cell Biol 2009; 186: 355-362.

23. Llambi F, Moldoveanu T, Tait SW, Bouchier-Hayes L, Temirov J, McCormick LL et al. A unified model of mammalian BCL-2 protein family interactions at the mitochondria. Mol cell 2011; 44: 517-531.

24. Gaudet S, Spencer SL, Chen WW, Sorger PK. Exploring the contextual sensitivity of factors that determine cell-to-cell variability in receptor-mediated apoptosis. PLoS Computational Biol 2012; 8: e1002482.

25. Garcia-Saez AJ, Ries J, Orzaez M, Perez-Paya E, Schwille P. Membrane promotes tBID interaction with BCL(XL). Nat Struct Mol Biol 2009; 16: 1178-1185.

26. Lovell JF, Billen LP, Bindner S, Shamas-Din A, Fradin C, Leber B et al. Membrane binding by tBid initiates an ordered series of events culminating in membrane permeabilization by Bax. Cell 2008; 135: 1074-1084.

27. Tait SWG, Green DR. Mitochondria and cell death: outer membrane permeabilization and beyond. Nat Rev Mol Cell Biol 2010; 11: 621-632.

28. Bender T, Martinou JC. Where killers meet-permeabilization of the outer mitochondrial membrane during apoptosis. Cold Spring Harbor Perspect Biol 2013; 5: a011106.

29. Todt F, Cakir Z, Reichenbach F, Youle RJ, Edlich F. The C-terminal helix of $\mathrm{Bcl}-\mathrm{x}(\mathrm{L})$ mediates Bax retrotranslocation from the mitochondria. Cell Death Differ 2013; 20: 333-342.

30. Edlich F, Banerjee S, Suzuki M, Cleland MM, Arnoult D, Wang C et al. Bcl-x(L) retrotranslocates Bax from the mitochondria into the cytosol. Cell 2011; 145: 104-116.

31. Kang R, Zeh HJ, Lotze MT, Tang D. The Beclin 1 network regulates autophagy and apoptosis. Cell Death Differ 2011; 18: 571-580.

32. Kilbride SM, Prehn JH. Central roles of apoptotic proteins in mitochondrial function. Oncogene 2013; 32: 2703-2711.

33. Autret A, Martin SJ. Bcl-2 family proteins and mitochondrial fission/fusion dynamics. Cell Mol Life Sci 2010; 67: 1599-1606.

34. Huber HJ, Dussmann H, Kilbride SM, Rehm M, Prehn JH. Glucose metabolism determines resistance of cancer cells to bioenergetic crisis after cytochrome-c release. Mol Syst Biol 2011; $7: 470$.

35. Taylor RC, Cullen SP, Martin SJ. Apoptosis: controlled demolition at the cellular level. Nat Rev Mol Cell Biol 2008; 9: 231-241.

36. Hellwig CT, Passante $E$, Rehm M. The molecular machinery regulating apoptosis signal transduction and its implication in human physiology and pathophysiologies. Curr Mol Med 2011; $11: 31-47$.

37. Tyas L, Brophy VA, Pope A, Rivett AJ, Tavare JM. Rapid caspase-3 activation during apoptosis revealed using fluorescence-resonance energy transfer. EMBO Rep 2000; 1 : 266-270.

38. Schmid J, Dussmann H, Boukes GJ, Flanagan L, Lindner AU, O'Connor CL et al. Systems analysis of cancer cell heterogeneity in caspase-dependent apoptosis subsequent to mitochondrial outer membrane permeabilization. J Biol Chem 2012; 287: 41546-41559.

39. Rehm M, Huber HJ, Dussmann H, Prehn JH. Systems analysis of effector caspase activation and its control by X-linked inhibitor of apoptosis protein. Embo $\mathrm{J} 2006$; 25: 4338-4349.

40. Srinivasula SM, Hegde R, Saleh A, Datta P, Shiozaki E, Chai J et al. A conserved XIAPinteraction motif in caspase- 9 and Smac/DIABLO regulates caspase activity and apoptosis. Nature 2001; 410: 112-116.

41. Legewie S, Bluthgen N, Herzel H. Mathematical modeling identifies inhibitors of apoptosis as mediators of positive feedback and bistability. PLoS Comput Biol 2006; 2: e120.

42. Zou H, Yang R, Hao J, Wang J, Sun C, Fesik SW et al. Regulation of the Apaf-1/caspase-9 apoptosome by caspase-3 and XIAP. J Biol Chem 2003; 278: 8091-8098.

43. Potts PR, Singh S, Knezek M, Thompson CB, Deshmukh M. Critical function of endogenous XIAP in regulating caspase activation during sympathetic neuronal apoptosis. J Cell Biol 2003; 163: 789-799.

44. Wright KM, Linhoff MW, Potts PR, Deshmukh M. Decreased apoptosome activity with neuronal differentiation sets the threshold for strict IAP regulation of apoptosis. J Cell Biol 2004; 167: 303-313.

45. Potts MB, Vaughn AE, McDonough H, Patterson C, Deshmukh M. Reduced Apaf-1 levels in cardiomyocytes engage strict regulation of apoptosis by endogenous XIAP. J Cell Biol 2005; 171: 925-930.

46. Albeck JG, Burke JM, Aldridge BB, Zhang M, Lauffenburger DA, Sorger PK. Quantitative analysis of pathways controlling extrinsic apoptosis in single cells. Mol Cell 2008; 30 : 11-25.

47. Nakabayashi J, Sasaki A. A mathematical model for apoptosome assembly: the optimal cytochrome c/Apaf-1 ratio. J Theor Biol 2006; 242: 280-287.
48. Ryu S, Lin SC, Ugel N, Antoniotti M, Mishra B. Mathematical modeling of the formation of apoptosome in intrinsic pathway of apoptosis. Systems and synthetic biology 2008; 2 : 49-66.

49. Kim J, Parrish AB, Kurokawa M, Matsuura K, Freel CD, Andersen JL et al. Rsk-mediated phosphorylation and 14-3-3varepsilon binding of Apaf-1 suppresses cytochrome c-induced apoptosis. EMBO J 2012; 31: 1279-1292.

50. Dhillon AS, Hagan S, Rath O, Kolch W. MAP kinase signalling pathways in cancer. Oncogene 2007; 26: 3279-3290.

51. Liontos M, Koutsami M, Sideridou M, Evangelou K, Kletsas D, Levy B et al. Deregulated overexpression of hCdt1 and hCdc6 promotes malignant behavior. Cancer Res 2007; 67: 10899-10909.

52. Gonzalez S, Klatt P, Delgado S, Conde E, Lopez-Rios F, Sanchez-Cespedes M et al. Oncogenic activity of Cdc6 through repression of the INK4/ARF locus. Nature 2006; 440: 702-706.

53. Allera-Moreau C, Rouquette I, Lepage B, Oumouhou N, Walschaerts M, Leconte E et al. DNA replication stress response involving PLK1, CDC6, POLQ, RAD51 and CLASPIN upregulation prognoses the outcome of early/mid-stage non-small cell lung cancer patients. Oncogenesis 2012; 1: e30.

54. Niimi S, Arakawa-Takeuchi S, Uranbileg B, Park JH, Jinno S, Okayama H. Cdc6 protein obstructs apoptosome assembly and consequent cell death by forming stable complexes with activated Apaf-1 molecules. J Biol Chem 2012; 287: 18573-18583.

55. Pelizon C, d'Adda di Fagagna F, Farrace L, Laskey RA. Human replication protein Cdc6 is selectively cleaved by caspase 3 during apoptosis. EMBO reports 2002; 3 : 780-784.

56. Malladi S, Challa-Malladi M, Fearnhead HO, Bratton SB. The Apaf- $1^{*}$ procaspase- 9 apoptosome complex functions as a proteolytic-based molecular timer. EMBO J 2009; 28: 1916-1925.

57. Saikumar P, Mikhailova M, Pandeswara SL. Regulation of caspase-9 activity by differential binding to the apoptosome complex. Front Biosci 2007; 12: 3343-3354.

58. Huber HJ, Laussmann MA, Prehn JH, Rehm M. Diffusion is capable of translating anisotropic apoptosis initiation into a homogeneous execution of cell death. BMC Systems Biol 2010; 4: 9 .

59. Williams DW, Kondo S, Krzyzanowska A, Hiromi Y, Truman JW. Local caspase activity directs engulfment of dendrites during pruning. Nat Neurosci 2006; 9: 1234-1236.

60. D'Amelio M, Cavallucci V, Middei S, Marchetti C, Pacioni S, Ferri A et al. Caspase-3 triggers early synaptic dysfunction in a mouse model of Alzheimer's disease. Nat Neurosci 2011; 14: 69-76.

61. Hanahan D, Weinberg RA. Hallmarks of cancer: the next generation. Cell 2011; 144 646-674.

62. Ni Chonghaile T, Sarosiek KA, Vo TT, Ryan JA, Tammareddi A, Moore Vdel G et al. Pretreatment mitochondrial priming correlates with clinical response to cytotoxic chemotherapy. Science 2011; 334: 1129-1133

63. Vo TT, Ryan J, Carrasco R, Neuberg D, Rossi DJ, Stone RM et al. Relative mitochondrial priming of myeloblasts and normal HSCs determines chemotherapeutic success in AML. Cell 2012; 151: 344-355.

64. Hector S, Rehm M, Schmid J, Kehoe J, McCawley N, Dicker P et al. Clinical application of a systems model of apoptosis execution for the prediction of colorectal cancer therapy responses and personalisation of therapy. Gut 2012; 61: 725-733.

65. Murphy AC, Weyhenmeyer B, Schmid J, Kilbride SM, Rehm M, Huber HJ et al. Activation of executioner caspases is a predictor of progression-free survival in glioblastoma patients: a systems medicine approach. Cell Death Disease 2013; 4: e629.

66. Passante E, Wurstle ML, Hellwig CT, Leverkus M, Rehm M. Systems analysis of apoptosis protein expression allows the case-specific prediction of cell death responsiveness of melanoma cells. Cell Death Differ 2013; 20: 1521-1531.

67. Poste G. Bring on the biomarkers. Nature 2011; 469: 156-157.

68. van den Boorn JG, Hartmann G. Turning tumors into vaccines: co-opting the innate immune system. Immunity 2013; 39: 27-37.

69. Kaczmarek A, Vandenabeele P, Krysko DV. Necroptosis: the release of damageassociated molecular patterns and its physiological relevance. Immunity 2013; 38 209-223.

70. Huang Q, Li F, Liu X, Li W, Shi W, Liu FF et al. Caspase 3-mediated stimulation of tumor cell repopulation during cancer radiotherapy. Nat Med 2011; 17: 860-866.

71. Li F, Huang Q, Chen J, Peng Y, Roop DR, Bedford JS et al. Apoptotic cells activate the "phoenix rising" pathway to promote wound healing and tissue regeneration. Sci Signal 2010; 3: ra13.

72. Chen J, Li Y, Yu TS, McKay RM, Burns DK, Kernie SG et al. A restricted cell population propagates glioblastoma growth after chemotherapy. Nature 2012; 488: 522-526.

73. Driessens G, Beck B, Caauwe A, Simons BD, Blanpain C. Defining the mode of tumour growth by clonal analysis. Nature 2012; 488: 527-530.

74. Sottoriva A, Vermeulen L, Tavare S. Modeling evolutionary dynamics of epigenetic mutations in hierarchically organized tumors. PLoS Computational Biol 2011; 7: e1001132.

75. Gentry SN, Jackson TL. A mathematical model of cancer stem cell driven tumor initiation: implications of niche size and loss of homeostatic regulatory mechanisms. PloS One 2013; 8: e71128.

76. Eissing T, Kuepfer L, Becker C, Block M, Coboeken K, Gaub T et al. A computational systems biology software platform for multiscale modeling and simulation: integrating 
whole-body physiology, disease biology, and molecular reaction networks. Front Physiol 2011; 2 : 4 .

77. Gorelik B, Ziv I, Shohat R, Wick M, Hankins WD, Sidransky D et al. Efficacy of weekly docetaxel and bevacizumab in mesenchymal chondrosarcoma: a new theranostic method combining xenografted biopsies with a mathematical model. Cancer Res 2008; 68: 9033-9040.

78. Kogan Y, Halevi-Tobias K, Elishmereni M, Vuk-Pavlovic S, Agur Z. Reconsidering the paradigm of cancer immunotherapy by computationally aided real-time personalization. Cancer Res 2012; 72: 2218-2227.

79. Arrowsmith J. Trial watch: phase II failures: 2008-2010. Nat Rev Drug Discov 2011; 10 328-329.

80. Arrowsmith J. Trial watch: phase III and submission failures: 2007-2010. Nat Rev Drug Discov 2011; 10: 87

81. Paul SM, Mytelka DS, Dunwiddie CT, Persinger CC, Munos BH, Lindborg SR et al. How to improve R\&D productivity: the pharmaceutical industry's grand challenge. Nat Rev Drug Discov 2010; 9: 203-214

82. Albeck JG, MacBeath G, White FM, Sorger PK, Lauffenburger DA, Gaudet S. Collecting and organizing systematic sets of protein data. Nat Rev Mol Cell Biol 2006; 7: 803-812.
83. Bentele M, Lavrik I, Ulrich M, Stosser S, Heermann DW, Kalthoff $\mathrm{H}$ et al. Mathematical modeling reveals threshold mechanism in CD95-induced apoptosis. J Cell Biol 2004; 166: 839-851.

84. Schleich K, Warnken U, Fricker N, Ozturk S, Richter P, Kammerer K et al. Stoichiometry of the CD95 death-inducing signaling complex: experimental and modeling evidence for a death effector domain chain model. Mol Cell 2012; 47: 306-319.

85. Gerdes MJ, Sevinsky CJ, Sood A, Adak S, Bello MO, Bordwell A et al. Highly multiplexed single-cell analysis of formalin-fixed, paraffin-embedded cancer tissue. Proc Natl Acad Sci USA 2013; 110: 11982-11987.

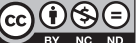

Cell Death and Disease is an open-access journal published by Nature Publishing Group. This work is licensed under a Creative Commons Attribution-NonCommercialNoDerivs 3.0 Unported License. To view a copy of this license, visit http://creativecommons.org/licenses/by-nc-nd/3.0/ 